\title{
Malignant Accessory Urethral Gland Neoplasm
}

National Cancer Institute

\section{Source}

National Cancer Institute. Malignant Accessory Urethral Gland Neoplasm. NCI

Thesaurus. Code C3561.

A malignant neoplasm involving the accessory urethral glands. 